\title{
Proof-relevance of families of setoids and identity in type theory
}

\author{
Erik Palmgren *
}

October 13, 2010; revised September 29, 2011

\begin{abstract}
Families of types are fundamental objects in Martin-Löf type theory. When extending the notion of setoid (type with an equivalence relation) to families of setoids, a choice between proof-relevant or proof-irrelevant indexing appears. It is shown that a family of types may be canonically extended to a proof-relevant family of setoids via the identity types, but that such a family is in general proofirrelevant if, and only if, the proof-objects of identity types are unique. A similar result is shown for fibre representations of families. The ubiquitous role of proofirrelevant families is discussed.
\end{abstract}

\section{Introduction}

This article investigates an aspect of the concept of extensional family of setoids - proof relevance - which sometimes presents a surprising obstacle when trying to transfer formalisations from set theory to standard Martin-Löf type theory [11]; compare Example 2.1 below. In set theory, the notion of a family of sets may readily be reduced to the notion of set. A family of sets may be represented as the fibres of a function $\beta: B \rightarrow A$. Its fibres $B_{x}=\beta^{-1}(x)=\{b \in B: \beta(b)=x\}$, for $x \in A$, represent the sets of the family. This representation is always possible in systems such as ZF, or in its constructive versions [1], since by the replacement scheme, any family specified by a set-theoretic formula $\varphi(x, F)$

$$
(\forall x \in A)(\exists ! F) \varphi(x, F)
$$

can be turned into a family represented by fibres of a function, i.e. there is a function $\beta: B \rightarrow A$ so that $(\forall x \in A) \varphi\left(x, \beta^{-1}(x)\right)$. This can be contrasted to Martin-Löf type

\footnotetext{
${ }^{*}$ Supported by a grant from the Swedish Research Council (VR). Research reported here was partly done during a stay at Institut Mittag-Leffler. The author is grateful for comments by anonymous referees that helped improve the presentation.
} 
theory [11], and other theories of dependent types, where a family of types is a basic mathematical object. Following the tradition in constructive mathematics (see [3]) a set is commonly understood in type theory as a setoid, that is, a type together with an equivalence relation. However the notion of a family of setoids present some choices for conceptualisation. In this note we consider two choices, so-called proof-irrelevant and proof-relevant families (see [4]), and their relation to the identity types of Martin-Löf. As shown by Streicher [12] and Hofmann and Streicher [7] an important distinction regarding identity types is whether their proof-objects are unique or not. In the former case a proof-irrelevant family of setoids can always be associated to each family of types. In the latter case a more involved proof-relevant notion of family of setoids seems be necessary to use; see Theorems 4.1 and 4.3. The distinction between proof-relevant and proof-irrelevant does not appear in classical set-theoretic models of Martin-Löf type theory, in view of a result by Hedberg [6] on uniqueness of identity proof-objects (UIP). We present a slight strengthening of his result in Section 6. Streicher introduced a computational axiomatization of UIP, the K-axiom. We discuss an alternative to this axiom in Section 7.

In this paper, the results can all be read as theorems of Martin-Löf type theory, given some standard conventions about the propositions-as-types interpretation [11]. By a type (or equivalently a proposition) $A$ being true, we mean that there is a construction or a proof-object $p$ such that the judgement $p: A$ is derivable. More generally, in a context

$$
A\left(x_{1}, \ldots, x_{n}\right) \text { true } \quad\left(x_{1}: X_{1}, \ldots, x_{n}: X_{n}\right)
$$

means there is a construction $p$ such that

$$
p\left(x_{1}, \ldots, x_{n}\right): A\left(x_{1}, \ldots, x_{n}\right) \quad\left(x_{1}: X_{1}, \ldots, x_{n}: X_{n}\right) \text {. }
$$

If no confusion is likely to arise we drop "true" from " $A$ true". We use the standard interpretation of quantifier and connectives in type theory, thus $\forall$ and $\exists$ are interpreted by $\Pi$ respectively $\Sigma$ etc.

\section{Families of setoids}

A setoid $B=\left(|B|,{ }_{B}\right)$ consists of a type $|B|$ and an equivalence relation, $={ }_{B}$, on the type. In the type-theoretic formalization, which we have in mind here, it is a 5-tuple, with proof-objects for reflexivity, symmetry and transitivity included in the definition. An extensional function between two setoids $B \rightarrow C$ is taken to be a function $f:|B| \longrightarrow|C|$ between underlying types that respects the equivalence relations. In a formalization the proof-object for extensionality $(\forall x, y:|B|)\left(x={ }_{B} y \longrightarrow f(x)={ }_{C} f(y)\right)$ is made explicit. Two such functions $f, g: B \longrightarrow C$ are then extensionally equal, $f={ }_{\text {ext }} g$, iff $(\forall x:|B|) f(x)={ }_{C} g(x)$. 
If $B_{x}=\left(\left|B_{x}\right|,=_{B_{x}}\right)$ are setoids indexed by a type $x:|A|$, there seems to be two principal choices how to extend this into a family indexed by a setoid $A=\left(|A|,{ }_{A}\right)$. Suppose the type $|A|$ is equipped with an equivalence relation, ${ }_{A}$. Each proof $p: x={ }_{A}$ $y$ should give rise to an extensional "reindexing" bijection $\phi_{p}: B_{x} \rightarrow B_{y}$. Starting from a set-theoretic intuition it is natural to require the following equalities of extensional functions to hold (see [3, Problem 3.2])

(i) $\phi_{p}={ }_{\text {ext }} \operatorname{id}_{B_{x}}$ whenever $p:\left(x={ }_{A} x\right)$,

(ii) $\phi_{q} \circ \phi_{p}={ }_{\text {ext }} \phi_{r}$, whenever $p:\left(x={ }_{A} y\right), q:\left(y={ }_{A} z\right), r:\left(x={ }_{A} z\right)$.

Together the two conditions (i) and (ii), implies that the proof $p$ is irrelevant, only its existence matter, i.e. $\phi_{p}={ }_{\mathrm{ext}} \phi_{q}$ for any $p: x=_{A} y$ and $q: x={ }_{A} y$. This is the standard version of family of setoid, and it seems to be the most widely used notion. The other principal version is the proof-relevant family, where $\phi_{p}$ may depend on $p$. Conditions (i) and (ii) are replaced by the weaker conditions (a) - (d):

(a) $\phi_{\operatorname{ref}(x)}={ }_{\text {ext }} \operatorname{id}_{B_{x}}$

(b) $\phi_{\operatorname{trans}(q, p)}={ }_{\text {ext }} \phi_{q} \circ \phi_{p}$ for $p: x={ }_{A} y$ and $q: y={ }_{A} z$

(c) $\phi_{\mathrm{sym}(p)} \circ \phi_{p}=\operatorname{ext}_{B_{x x}}$ for $p: x={ }_{A} y$,

(d) $\phi_{p} \circ \phi_{\mathrm{sym}(p)}=\operatorname{ext}_{\mathrm{id}_{B_{y}}}$ for $p: x={ }_{A} y$.

Here $\operatorname{ref}(x): x={ }_{A} x$ is a proof object for reflexivity. Moreover the proof objects associated with symmetry and transitivity are $\operatorname{sym}(p): y={ }_{A} x$, for $p: x={ }_{A} y$, and $\operatorname{trans}(q, p): x={ }_{A} z$ for $p: x={ }_{A} y$ and $q: y={ }_{A} z$.

We note that $\phi_{p}: B_{x} \rightarrow B_{x}$ is an automorphism on $B_{x}$ for each $p: x={ }_{A} x$. There may be non-trivial automorphisms, i.e. other than the identity map. However, if we add the proof-irrelevance condition

(Irr) $\phi_{p}={ }_{\text {ext }} \phi_{q}$ for any $p: x={ }_{A} y$ and $q: x={ }_{A} y$.

to (a) - (d) then (i) and (ii) follows, and clearly there are then only trivial automorphisms. A family of setoids is called proof irrelevant if it satisfies (a) $-(\mathrm{d})+(\operatorname{Irr})$, or equivalently (i) and (ii).

Example 2.1 As an example of the desirability of the standard proof-irrelevant version of family, we consider the problem of constructing certain categories of setoids and functions. This occurs for in particular when we wish to define a small category of setoids using a type universe.

Let $(B, \phi)$ be a given family of setoids indexed by the setoid $A$. We construct a small category $\mathcal{B}$ from this data. The collection of objects of the category $\mathcal{B}$ is the setoid $A$. We think of an element $a$ of $A$ as a code for the setoid $B_{a}$. An arrow of the 
category is a triple $(a, f, b)$ where $a, b:|A|$ and $f: B_{a} \longrightarrow B_{b}$ is an extensional function. Two arrows $(a, f, b)$ and $\left(a^{\prime}, f^{\prime}, b^{\prime}\right)$ are equivalent if there are $p: a={ }_{A} a^{\prime}$ and $q: b={ }_{A} b^{\prime}$ so that

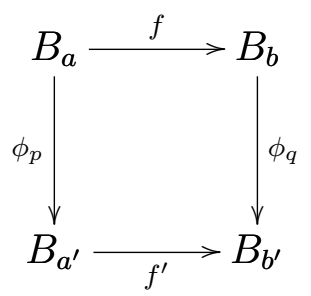

commutes (extensionally). Arrows $(a, f, b)$ and $(c, g, d)$ are composable if there is $t$ : $b={ }_{A} c$. Their composition is $\left(a, g \circ \phi_{t} \circ f, d\right)$. Now a problem arises when proving that composition respects equality of arrows: suppose that $(a, b, f)$ and $\left(a^{\prime}, b^{\prime}, f^{\prime}\right)$ are equivalent, and that $(c, g, d)$ and $\left(c^{\prime}, g^{\prime}, d^{\prime}\right)$ are equivalent so that the left and right squares in the following diagram commute:

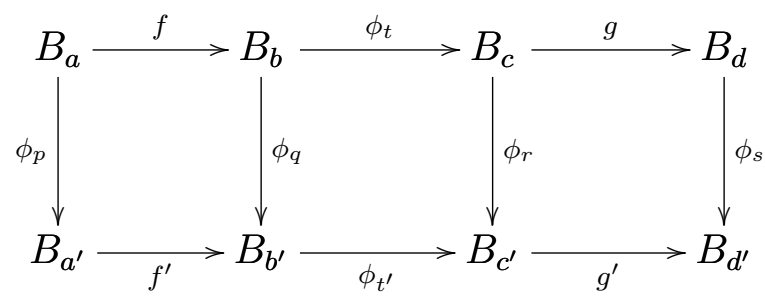

Suppose moreover that $t: b={ }_{A} c$ and $t^{\prime}: b^{\prime}={ }_{A} c^{\prime}$. If $(B, \phi)$ is a proof-irrelevant family the centre square commutes automatically, proving that composition respects equality of arrows. This is in general false if the family is proof-relevant.

\section{Identity types}

The presentation of Martin-Löf type theory given in [11] will be followed here, but we shall use the older terminology of type for what is now called a set, and large type or Type for what is now called just type. The identity type construction provides for each type $A$ a minimal equivalence relation $\mathrm{I}(A, \cdot, \cdot)$ on $A$. This makes $(A, \mathrm{I}(A, \cdot, \cdot))$ a projective object (cf. [9]) in the category of setoids.

For any type $A$, the identity type $\mathrm{I}(A, a, b)$ is the set of proofs that $a$ and $b$ are propositionally equal in $A$. The formation rule for the identity type is that $\mathrm{I}(A, a, b)$ is a type whenever $A$ is a type and $a, b: A$. We shall also write $\mathrm{I}_{A}(a, b)$, or even $\mathrm{I}(a, b)$, for $\mathrm{I}(A, a, b)$ if this appears to be typographically clearer. The introduction rule is

$$
\frac{a: A}{\mathrm{r}(a): \mathrm{I}(A, a, a)}
$$


The elimination rule for $\mathrm{I}$ with respect to the family $C(x, y, z)$ type $(x, y: A, z$ : $\mathrm{I}(A, x, y))$ is

$$
\frac{a, b: A \quad c: \mathrm{I}(A, a, b) \quad d(x): C(x, x, r(x))(x: A)}{\mathrm{J}_{C, a, b}(c, d): C(a, b, c)} .
$$

The associated computation rule is $\mathrm{J}_{C, a, a}(\mathrm{r}(a), d)=d(a)$. A typical application is to derive a rule for substituting equals for equals in a proposition, or equivalently, derive a reindexing operation for families. For $B(x)$ type $(x: A)$, define $C(x, y, z)=B(x) \rightarrow$ $B(y)$. Then $d(x)=\operatorname{id}_{B(x)}=\lambda p: B(x) \cdot p: C(x, x, \mathrm{r}(x))$. Hence for $c: \mathrm{I}(A, a, b)$

$$
\mathrm{J}_{C, a, b}\left(c,(x) \operatorname{id}_{B(x)}\right): C(a, b, c)=B(a) \rightarrow B(b) .
$$

Define

$$
\mathrm{R}_{B, a, b}(c, q)=\mathrm{J}_{C, a, b}\left(c,(x) \operatorname{id}_{B(x)}\right)(q): B(b)
$$

for $q: B(a)$. Clearly $\mathrm{R}_{B, a, a}(\mathrm{r}(a), q)=q$.

A very useful elimination rule is the rule of Paulin-Mohring; see appendix of Streicher [12] for a proof that it is equivalent to the standard rule (1). It says that for any parameter $a: A$ and any family $D(x, z)$ type $(x: A, z: \mathrm{I}(A, a, x))$ if

$$
\frac{b: A \quad c: \mathrm{I}(A, a, b) \quad d: D(a, r(a))}{\mathrm{J}^{\prime}{ }_{a, D, b}(c, d): D(b, c)}
$$

The computation rule is $\mathrm{J}_{a, D, a}^{\prime}(r(a), d)=d$.

The identity proofs of $A$ are said to be unique in case

$$
\left(\forall z, w: \mathrm{I}_{A}(a, b)\right) \mathrm{I}(z, w) \quad\left(\mathrm{UIP}_{A}\right)
$$

holds. We say that UIP holds if for each type $A$ satisfies $\mathrm{UIP}_{A}$. Hofmann and Streicher [7] showed that this need not hold for general types by exhibiting a groupoid model of type theory. The structure of identity types thus turned out to be more complicated than expected. In fact, they showed that it induces a groupoid structure on each type, as follows. Using the standard elimination rule (1) one constructs operations for proofs of symmetry and transitivity

$$
\begin{aligned}
& c^{-1}: \mathrm{I}(A, b, a) \quad(a, b: A, c: \mathrm{I}(A, a, b)), \\
& \text { where } c^{-1}=\mathrm{J}_{C, a, b}(c, \mathrm{r}) \text { and } C(x, y, z)=\mathrm{I}(A, y, x) \text {, } \\
& w \circ z: \mathrm{I}(A, a, u) \quad(a, b, u: A, z: \mathrm{I}(A, a, b), w: \mathrm{I}(A, b, u)), \\
& \text { where } w \circ z=\mathrm{J}_{C, a, b}(z, d)(w), C\left(x, y, z^{\prime}\right)=\mathrm{I}(A, y, u) \rightarrow \mathrm{I}(A, x, u) \text { and } d(x)= \\
& \operatorname{id}_{\mathrm{I}(A, x, u)} \text {. }
\end{aligned}
$$


These operations satisfy the groupoid laws with $1_{x}={ }_{\text {def }} \mathrm{r}(x)$ as identity in the sense that the following identity statements hold:

(G1) $\mathrm{I}\left(1_{y} \circ z, z\right)$ for $z: \mathrm{I}(A, x, y)$,

(G2) $\mathrm{I}\left(z \circ 1_{x}, z\right)$ for $z: \mathrm{I}(A, x, y)$,

(G3) $\mathrm{I}\left(z \circ z^{-1}, 1_{y}\right)$ for $z: \mathrm{I}(A, x, y)$,

(G4) $\mathrm{I}\left(z^{-1} \circ z, 1_{x}\right)$ for $z: \mathrm{I}(A, x, y)$,

(G5) $\mathrm{I}((z \circ w) \circ p, z \circ(w \circ p))$ for $p: \mathrm{I}(A, x, y), w: \mathrm{I}(A, y, u), z: \mathrm{I}(A, u, v)$.

The type-theoretic version of a groupoid is an E-category [2] where all morphisms are invertible. To be explicit: A groupoid $A=\left(|A|, \operatorname{Hom}, 1, \circ,()^{-1}\right)$ consists of

- a type $|A|$,

- a setoid $\operatorname{Hom}(a, b)$ of morphisms for any $a, b:|A|$,

- an identity morphism $1_{a} \in \operatorname{Hom}(a, a)$ for each $a:|A|$,

- a composition operation $\circ: \operatorname{Hom}(b, c) \times \operatorname{Hom}(a, b) \longrightarrow \operatorname{Hom}(a, c)$ for all $a, b, c:|A|$,

- an inversion ()$^{-1}: \operatorname{Hom}(a, b) \longrightarrow \operatorname{Hom}(b, a)$ for $a, b:|A|$,

satisfying the groupoid laws up to the equality of the Hom-setoids. From (G1-G5) above follows that each type $A$ yields a groupoid $A^{\star}=\left(A, \operatorname{Hom}, \mathrm{id}, \circ,()^{-1}\right)$ where $\operatorname{Hom}(a, b)=\left(\mathrm{I}(A, a, b), \mathrm{I}_{\mathrm{I}(A, a, b)}(\cdot, \cdot)\right)$.

\section{Families of setoids induced by families of types}

Any family $B$ of types over $A$, i.e. a type-valued function $B:(A)$ type in the notation of [11], gives rise to a proof-relevant family of setoids. Define $A^{*}=(A, \mathrm{I}(A, \cdot, \cdot))$ and $B^{*}(a)=(B(a), \mathrm{I}(B(a), \cdot, \cdot))$ and define $\phi_{p}: B(a) \rightarrow B(b)$, by $\phi_{p}(x)=\mathrm{R}_{B, a, b}(p, x)$. The reindexing operation $\mathrm{R}$ of $(3)$ is functorial in the sense that

(R1) $\mathrm{I}\left(\mathrm{R}_{B, a, a}(\mathrm{r}(a), w), w\right)$ holds for $a: A, w: B(a)$,

(R2) $\mathrm{I}\left(\mathrm{R}_{B, b, c}\left(t, \mathrm{R}_{B, a, b}(z, w)\right), \mathrm{R}_{B, a, c}((t \circ z), w)\right)$ holds for $a, b, c: A$ and $z: \mathrm{I}(A, a, b)$ and $t: \mathrm{I}(A, b, c)$ and $w: B(a)$.

We shall also write $B_{p}^{*}$ for $\phi_{p}$. I-elimination gives

$$
\mathrm{I}(\mathrm{I}(A, a, b), p, q) \Longrightarrow B_{p}^{*}={ }_{\text {ext }} B_{q}^{*} .
$$

The groupoid laws G1 - G5 gives with $\operatorname{ref}(\mathrm{x})=\mathrm{id}_{x}, \operatorname{sym}(p)=p^{-1}$ and $\operatorname{trans}(q, p)=q \circ p$ the following theorem: 
Theorem 4.1 For any family of types $B:(A)$ type the construction $\left(A^{*}, B^{*}\right)$ is a proofrelevant family of setoids.

We show that UIP gives a precise condition on the index setoid of this family, in order for the family to be proof-irrelevant. For this we use that, in a special case, the reindexing operation is a composition operation:

Lemma 4.2 For $u: A$ and $B(x)=\mathrm{I}(A, u, x)$, it holds that

$$
\mathrm{I}\left(\mathrm{R}_{B, a, b}(z, v), z \circ v\right)
$$

for $z: \mathrm{I}(A, a, b)$ and $v: \mathrm{I}(A, u, a)$.

Proof. Let $C(a, b, z)$ be the formula

$$
(\forall v: \mathrm{I}(A, u, a)) \mathrm{I}_{B(b)}\left(\mathrm{R}_{B, a, b}(z, v), z \circ v\right) .
$$

Now $C(a, a, r(a))$ is

$$
(\forall v: \mathrm{I}(A, u, a)) \mathrm{I}_{B(a)}\left(\mathrm{R}_{B, a, a}(\mathrm{r}(a), v), \mathrm{r}(a) \circ v\right),
$$

which by the groupoid laws and $\mathrm{R}_{B, a, a}(\mathrm{r}(a), q)=q$ is equivalent to

$$
(\forall v: \mathrm{I}(A, u, a)) \mathrm{I}_{B(a)}(v, v) .
$$

But this follows by the reflexivity law, so by I-elimination $C(a, b, z)$ is true. Hence the lemma is proved.

Theorem 4.3 Let $A$ be a fixed type. Then UIP holds for $A$ if and only if $\left(A^{*}, B^{*}\right)$ is a proof-irrelevant family of setoids, for any family $B$ : (A)type.

Proof. In view of Theorem 4.1 we may concentrate on the condition (Irr) for proofirrelevance.

$(\Rightarrow)$ : Assume that UIP holds for $A$. For $p, p^{\prime}: \mathrm{I}(A, a, b)$ there is $c: \mathrm{I}\left(\mathrm{I}(A, a, b), p, p^{\prime}\right)$. Let

$$
C(u, v, z)=(\forall x: B(a))\left(B_{u}^{*}(x)={ }_{B(b)} B_{v}^{*}(x)\right),
$$

where $u, v: \mathrm{I}(A, a, b)$. Clearly, $C(u, u, \mathrm{r}(u))$ is inhabited since $=_{B(b)}$ is reflexive. Hence by the elimination rule for $I$, we get that $C\left(p, p^{\prime}, c\right)$ is true, which says that $B^{*}$ is proof irrelevant.

$(\Leftarrow)$ : Suppose that $\left(A^{*}, B^{*}\right)$ is a proof-irrelevant family of setoids, for any $B$ : (A)type. Fix $a: A$, and let $B(x)=\mathrm{I}(A, a, x)$. Then condition (Irr) for $B^{*}$ is that

$$
\mathrm{I}\left(\mathrm{I}(A, a, b), B_{p}^{*}(q), B_{p^{\prime}}^{*}(q)\right)
$$

holds for $a, b: A, p, p^{\prime}: \mathrm{I}(A, a, b), q: B(a)=\mathrm{I}(A, a, a)$. Now by Lemma 4.2 and $B_{p}^{*}(q)=\mathrm{R}_{B, a, b}(p, q)$ the equation (6) is equivalent to

$$
\mathrm{I}\left(\mathrm{I}(A, a, b), p \circ q, p^{\prime} \circ q\right) .
$$

Putting $q=\mathrm{r}(a)$, we get $\mathrm{I}\left(\mathrm{I}(A, a, b), p \circ \mathrm{r}(a), p^{\prime} \circ \mathrm{r}(a)\right)$, and since $\mathrm{r}(a)$ is an identity of the groupoid, we have $\mathrm{I}\left(\mathrm{I}(A, a, b), p, p^{\prime}\right)$ for all $p, p^{\prime}: \mathrm{I}(A, a, b)$. That is, UIP holds for A. 


\section{$5 \quad$ Families of setoids induced by fibres of maps.}

Analogous to set theory, we may present a family of setoids in type theory via fibres of a function $f: S \rightarrow A$ between setoids. Define the fibre of $f$ over $a$ as the setoid

$$
f^{-1}(a)={ }_{\operatorname{def}}\left((\Sigma z: S)\left(f(z)={ }_{A} a\right), \sim\right),
$$

where $(z, p) \sim\left(z^{\prime}, p^{\prime}\right)$ holds if and only if $z={ }_{S} z^{\prime}$. For $q: a={ }_{A} b$ let $f^{-1}(q): f^{-1}(a) \rightarrow$ $f^{-1}(b)$ be given by

$$
f^{-1}(q)(z, p)=(z, q \circ p) .
$$

This clearly defines a proof-irrelevant family of setoids.

Using the UIP it is possible to obtain each family $\left(A^{*}, B^{*}\right)$ as the fibres of a certain projection function $\pi_{1}: S \longrightarrow A^{*}$. For this we need a lemma about identity types.

Lemma 5.1 On a sigma type $S=(\Sigma x: A) B(x)$, the I-equality is characterised by

$$
\mathrm{I}\left(S,(a, b),\left(a^{\prime}, b^{\prime}\right)\right) \Longleftrightarrow\left(\exists p: \mathrm{I}\left(A, a, a^{\prime}\right)\right) \mathrm{I}\left(B\left(a^{\prime}\right), \mathrm{R}_{B, a, a^{\prime}}(p, b), b^{\prime}\right) .
$$

Proof. $(\Leftarrow)$ We show that $\left(\forall a, a^{\prime}: A\right)\left(\forall p: \mathrm{I}\left(A, a, a^{\prime}\right)\right) C\left(a, a^{\prime}, p\right)$ where

$$
C\left(a, a^{\prime}, p\right)=(\forall b: B(a))\left(\forall b^{\prime}: B\left(a^{\prime}\right)\right)\left[\mathrm{I}\left(B\left(a^{\prime}\right), \mathrm{R}_{B, a, a^{\prime}}(p, b), b^{\prime}\right) \rightarrow \mathrm{I}\left(S,(a, b),\left(a^{\prime}, b^{\prime}\right)\right)\right] .
$$

By I-elimination it suffices to show $C(a, a, \mathrm{r}(a))$ which using $\mathrm{R}_{B, a, a}(\mathrm{r}(a), b)=b$ is

$$
(\forall b: B(a))\left(\forall b^{\prime}: B(a)\right)\left[\mathrm{I}\left(B(a), b, b^{\prime}\right) \rightarrow \mathrm{I}\left(S,(a, b),\left(a, b^{\prime}\right)\right)\right] .
$$

But this follows directly by another application of I-elimination.

$(\Rightarrow)$ By $\Sigma$-elimination we find for $z: S$ terms $\pi_{1}(z): A$ and $\pi_{2}(z): B\left(\pi_{2}(z)\right)$ so that $\pi_{1}((a, b))=a$ and $\pi_{2}((a, b))=b$. For $z, z^{\prime}: S$ and $q: \mathrm{I}\left(S, z, z^{\prime}\right)$, let $C\left(z, z^{\prime}, q\right)$ be

$$
\left(\exists p: \mathrm{I}\left(A, \pi_{1}(z), \pi_{1}\left(z^{\prime}\right)\right)\right) \mathrm{I}\left(B\left(\pi_{1}\left(z^{\prime}\right)\right), \mathrm{R}_{B, \pi_{1}(z), \pi_{1}\left(z^{\prime}\right)}\left(p, \pi_{2}(z)\right), \pi_{2}\left(z^{\prime}\right)\right) .
$$

By I-elimination it is sufficient to prove $C(z, z, \mathrm{r}(z))$ for all $z: S$. By $\Sigma$-elimination it is enough that $C((a, b),(a, b), \mathrm{r}(a, b))$ holds, i.e.

$$
(\exists p: \mathrm{I}(A, a, a)) \mathrm{I}\left(B(a), \mathrm{R}_{B, a, a}(p, b), b\right) .
$$

This can be achieved by letting $p=\mathrm{r}(a)$ and using $\mathrm{R}_{B, a, a}(\mathrm{r}(a), b)=b$ and I-introduction. Consequently, if $q: \mathrm{I}\left(S,(a, b),\left(a^{\prime}, b^{\prime}\right)\right)$, then $C\left((a, b),\left(a^{\prime}, b^{\prime}\right), q\right)$ as was desired.

Theorem 5.2 Let $A$ be a fixed type. For a family $B:(A)$ type, let $S=(\Sigma x: A) B(x)$ and let $\pi_{1}: S^{*} \rightarrow A^{*}$ be the first projection. For each $a:$ A define $\theta_{a}: \pi_{1}^{-1}(a) \rightarrow B(a)^{*}$ by letting

$$
\theta_{a}((u, v), p)=\mathrm{R}_{B, u, a}(p, v) .
$$

Then $\theta_{a}$ is a well-defined bijection of setoids for any $a: A$ and any choice of $B:(A)$ type if and only if $A$ satisfies UIP. 
Proof. $(\Leftarrow)$ : Assume that $A$ satisfies UIP. Suppose $((u, v), p)$ and $\left(\left(u^{\prime}, v^{\prime}\right), p^{\prime}\right)$ are equal in $\pi_{1}^{-1}(a)$. Thus by definition $p: \mathrm{I}(A, u, a), p^{\prime}: \mathrm{I}\left(A, u^{\prime}, a\right)$ and $\mathrm{I}\left(S,(u, v),\left(u^{\prime}, v^{\prime}\right)\right)$ holds. By Lemma 5.1 follows that there is some $q: \mathrm{I}\left(A, u, u^{\prime}\right)$ with $\mathrm{I}\left(B\left(u^{\prime}\right), \mathrm{R}_{B, u, u^{\prime}}(q, v), v^{\prime}\right)$. Thus $\mathrm{I}\left(B(a), \mathrm{R}_{B, u^{\prime}, a}\left(p^{\prime},\left(\mathrm{R}_{B, u, u^{\prime}}(q, v)\right), \mathrm{R}_{B, u^{\prime}, a}\left(p^{\prime}, v^{\prime}\right)\right)\right.$. By the functoriality property (R2) it follows

$$
\mathrm{I}\left(B(a), \mathrm{R}_{B, u, a}\left(p^{\prime} \circ q, v\right), \mathrm{R}_{B, u^{\prime}, a}\left(p^{\prime}, v^{\prime}\right)\right) .
$$

But UIP for $A$ gives that $\mathrm{I}\left(A, p^{\prime} \circ q, p\right)$ holds, and hence by I-elimination we have that

$$
\mathrm{I}\left(B(a), \mathrm{R}_{B, u, a}(p, v), \mathrm{R}_{B, u^{\prime}, a}\left(p^{\prime}, v^{\prime}\right)\right)
$$

holds, thus proving $\theta_{a}$ well-defined. Suppose now (7) where $p: \mathrm{I}(A, u, a)$ and $p^{\prime}$ : $\mathrm{I}\left(A, u^{\prime}, a\right)$. Hence $\left(p^{\prime}\right)^{-1} \circ p: \mathrm{I}\left(A, u, u^{\prime}\right)$ and applying $\mathrm{R}\left(\left(p^{\prime}\right)^{-1}, \cdot\right)$ to $(7)$ we obtain by functoriality and (G4)

$$
\mathrm{I}\left(B(a), \mathrm{R}_{B, u, u^{\prime}}\left(\left(p^{\prime}\right)^{-1} \circ p, v\right), v^{\prime}\right) .
$$

Hence by Lemma 5.1,

$$
\mathrm{I}\left(S,(u, v),\left(u^{\prime}, v^{\prime}\right)\right)
$$

Thus $\theta_{a}$ is injective. To prove surjectivity, let $b: B(a)$ and consider $\theta_{a}((a, b), \mathrm{r}(a))=$ $\mathrm{R}_{B, a, a}(\mathrm{r}(a), b)=b$. Thus $\theta_{a}$ is a well-defined bijection.

$(\Rightarrow)$ : Suppose that $\theta_{a}$ is a well-defined bijection for each choice of $B$, and any $a: A$. Let $a, b: A$ be fixed. Define $B(x)=\mathrm{I}(A, a, x)$. Let $z: B(b)$ and $w$ : $B(b)$. Hence $p=w \circ z^{-1}: \mathrm{I}(A, b, b)$. Then $\mathrm{I}(B(b), p \circ z, w)$ holds by the groupoid laws. But $\mathrm{I}\left(B(b), \mathrm{R}_{B, b, b}(p, z), p \circ z\right)$ by Lemma 4.2 with $a=b$ and $u=a$, and hence $\mathrm{I}\left(B(b), \mathrm{R}_{B, b, b}(p, z), w\right)$. It follows by Lemma 5.1 that $\mathrm{I}(S,(b, z),(b, w))$, and hence that $((b, z), \mathrm{r}(b))$ and $((b, w), \mathrm{r}(b))$ are equal in $\pi_{1}^{-1}(b)$. Now $\theta_{b}$ is well-defined, so $\mathrm{I}\left(B(b), \theta_{b}((b, z), \mathrm{r}(b)), \theta_{b}((b, w), \mathrm{r}(b))\right)$ holds, i.e. $\mathrm{I}(B(b), z, w)=\mathrm{I}(\mathrm{I}(A, a, b), z, w)$ is inhabited.

Remark 5.3 This result shows that the statement that $\theta_{a}$ is a well-defined bijection in Moerdijk and Palmgren [10, p. 196, line $13-15]$ actually need the assumption UIP to be true. This assumption was omitted in that paper.

\section{$6 \quad$ Decidable identity types}

Hedberg [6] proved that decidable identity types satisfy UIP in the following sense:

Theorem 6.1 (Hedberg) If $(\forall x, y: A)\left(\mathrm{I}_{A}(x, y) \vee \neg \mathrm{I}_{A}(x, y)\right)$, then

$$
(\forall x, y: A)\left(\forall u, v: \mathrm{I}_{A}(x, y)\right) \mathrm{I}(u, v) .
$$

This result shows that UIP is always true in classical extensions of type theory. Examining the proof in [6] one can see that the same argument proves the somewhat stronger statement 
Theorem 6.2 Let $x: A$ be fixed. If $(\forall y: A)\left(\mathrm{I}_{A}(x, y) \vee \neg \mathrm{I}_{A}(x, y)\right)$, then

$$
(\forall y: A)\left(\forall u, v: \mathrm{I}_{A}(x, y)\right) \mathrm{I}(u, v) .
$$

Note that to apply this theorem one does not need to assume that $\mathrm{I}(A, x, y)$ is decidable for every pair $x$ and $y$. For instance, if $A$ is an infinitary tree, say given by the introduction rules

$$
0: A \quad \frac{f: N \rightarrow A}{\sup (f): A}
$$

we may not able to decide equality in general. However, for $x=0, \mathrm{I}(A, x, y)$ can be decided for all $y: A$, using the appropriate elimination rule.

The main ingredients of Hedberg's theorem are two lemmas, of which we modify the second.

Lemma 6.3 If $S \vee \neg S$, then there is $f: S \rightarrow S$ with

$$
(\forall x, y: S) \mathrm{I}_{S}(f(x), f(y))
$$

Proof. If $a: S$, then we may let $f(x)=a$. If $a: \neg S$, then take $f(x)=x$.

Lemma 6.4 Let $x: A$. If $f:(\Pi y: A)(\mathrm{I}(A, x, y) \rightarrow \mathrm{I}(A, x, y))$, then there is $g:(\Pi y$ : $A)(\mathrm{I}(A, x, y) \rightarrow \mathrm{I}(A, x, y))$ with

$$
(\forall y: A)\left(\forall z: \mathrm{I}_{A}(x, y)\right) \mathrm{I}(g(y, f(y, z)), z) .
$$

Proof. Employing the groupoid operations construct $g$ as follows

$$
g(y, w)=w \circ(f(x, r(x)))^{-1}
$$

for $y: A, w: \mathrm{I}(A, x, y)$. Instead of using the standard elimination rule as in [6], we shall use Paulin-Mohring's rule (4). Take $D(u, z)$ to be

$$
\mathrm{I}(\mathrm{I}(A, x, u), g(u, f(u, z)), z),
$$

where $u: A, z: \mathrm{I}(A, x, u)$. Now $D(x, r(x))$ is

$$
\mathrm{I}\left(\mathrm{I}(A, x, x), f(x, r(x)) \circ(f(x, r(x)))^{-1}, r(x)\right),
$$

which is true in virtue of the groupoid laws. Say the type is inhabited by the proof object $p$. Thus for any $y: A$ and $z: \mathrm{I}(A, x, y)$ we have that $\mathrm{J}_{x, D, y}^{\prime}(z, p): D(y, z)$. That is we have proved

$$
(\forall y: A)\left(\forall z: \mathrm{I}_{A}(x, y)\right) \mathrm{I}(g(y, f(y, z)), z) .
$$


Proof of Theorem 6.2. Let $x: A$ and suppose that $(\forall y: A)\left(\mathrm{I}_{A}(x, y) \vee \neg \mathrm{I}_{A}(x, y)\right)$. Thus by Lemma 6.3 we find for each $y: A, f(y): \mathrm{I}(A, x, y) \rightarrow \mathrm{I}(A, x, y)$ with

$$
\left(\forall z, w: \mathrm{I}_{A}(x, y)\right) \mathrm{I}(f(y, z), f(y, w)) .
$$

Lemma 6.4 gives $g:(\Pi y: A)(\mathrm{I}(A, x, y) \rightarrow \mathrm{I}(A, x, y))$ with

$$
(\forall y: A)\left(\forall z: \mathrm{I}_{A}(x, y)\right) \mathrm{I}(g(y, f(y, z)), z) .
$$

Thus applying $g$ to (8) we get for each $y: A$

$$
\left(\forall z, w: \mathrm{I}_{A}(x, y)\right) \mathrm{I}(g(y, f(y, z)), g(y, f(y, w))) .
$$

By (9) twice we obtain

$$
\left(\forall z, w: \mathrm{I}_{A}(x, y)\right) \mathrm{I}(z, w)
$$

\section{Axiomatizing uniqueness of identity proofs}

In order to axiomatize UIP, Streicher [12] suggested to supplement the standard elimination operator $\mathrm{J}$ for identity types with a second elimination operator $\mathrm{K}$ given by the rule: for $D(x, z)$ type $(x: A, z: \mathrm{I}(A, x, x))$

$$
\frac{c: \mathrm{I}(A, a, a) \quad d(x): D(x, \mathrm{r}(x)) \quad(x: A)}{\mathrm{K}_{D, a}(c, d): D(a, c)}
$$

and the computation rule $\mathrm{K}_{D, a}(\mathrm{r}(a), d)=d(a)$. This rule is akin to the elimination rule for the unit type - compare (12) below — in that it states that $\mathrm{I}(A, a, a)$ has exactly one element (namely $\mathrm{r}(a)$ ).

Though $\mathrm{K}$ has a good computational interpretation, having two elimination operators clearly fall outside the pattern of standard Martin-Löf type theory. We show here that the operators $\mathrm{J}$ and $\mathrm{K}$ may at least be combined into a single operator $\mathrm{J}^{2}$ given by the elimination rule: for $C(x, y, u, v)$ type $(x: A, y: A, u: \mathrm{I}(A, x, y), v: \mathrm{I}(A, x, y))$ we have

$$
\frac{c: \mathrm{I}(A, a, b) \quad c^{\prime}: \mathrm{I}(A, a, b) \quad d(x): C(x, x, \mathrm{r}(x), \mathrm{r}(x)) \quad(x: A)}{\mathrm{J}_{C, a, b}^{2}\left(c, c^{\prime}, d\right): C\left(a, b, c, c^{\prime}\right)}
$$

with computation rule $\mathrm{J}_{C, a, a}^{2}(\mathrm{r}(a), \mathrm{r}(a), d)=d(a)$. This can be considered as a sort of double recursion operator on identity types; see Remark 7.2 below.

Theorem 7.1 $\mathrm{J}^{2}$ is equivalent to the combination of $\mathrm{J}$ and $\mathrm{K}$. 
Proof. We show that these rules are interderivable.

$\left(\mathrm{J}+\mathrm{K} \Rightarrow \mathrm{J}^{2}\right)$ : Define $D(x, v)=C(x, x, \mathrm{r}(x), v) \quad(x: A, v: \mathrm{I}(A, x, x))$. Thus $d(x): D(x, \mathrm{r}(x)) \quad(x: A)$, so $\mathrm{K}_{D, x}(v, d): D(x, v)$ for $x: A$ and $v: \mathrm{I}(A, x, x)$ by the K-rule. Abbreviate $\mathrm{K}_{D, x}$ by $\mathrm{K}_{x}$. Define

$$
E(x, y, z)=(\Pi w: \mathrm{I}(A, x, y)) C(x, y, z, w) .
$$

Thus we have $\lambda v \cdot \mathrm{K}_{x}(v, d): E(x, x, \mathrm{r}(x))$. By the J-rule we have $\mathrm{J}_{E, a, b}\left(c,(x) \lambda v \cdot \mathrm{K}_{x}(v, d)\right)$ : $E(a, b, c)$. Thus define

$$
\mathrm{J}_{C, a, b}^{2}\left(c, c^{\prime}, d\right)=\mathrm{J}_{E, a, b}\left(c,(x) \lambda v \cdot \mathrm{K}_{x}(v, d)\right)\left(c^{\prime}\right): C\left(a, b, c, c^{\prime}\right) .
$$

Clearly, $\mathrm{J}_{C, a, a}^{2}(\mathrm{r}(a), \mathrm{r}(a), d)=\mathrm{J}_{E, a, a}\left(\mathrm{r}(a),(x) \lambda v \cdot \mathrm{K}_{x}(v, d)\right)(\mathrm{r}(a))=\mathrm{K}_{a}(\mathrm{r}(a), d)=d(a)$. This proves $\left(\mathrm{J}+\mathrm{K} \Rightarrow \mathrm{J}^{2}\right)$.

$\left(\mathrm{J}^{2} \Longrightarrow \mathrm{J}\right)$ : Suppose $E(x, y, z)$ type $\quad(x, y: A, z: \mathrm{I}(A, x, y))$ and $e(x): E(x, x, \mathrm{r}(x)) \quad(x:$ $A)$. Define $C(x, y, u, v)=E(x, y, u)$. Thus also $e(x): C(x, x, \mathrm{r}(x), \mathrm{r}(x)) \quad(x: A)$. For $c: \mathrm{I}(A, a, b)$ we have $\mathrm{J}_{C, a, b}^{2}(c, c, e): C(a, b, c, c)$. We define $\mathrm{J}_{E, a, b}(c, e)=\mathrm{J}_{C, a, b}^{2}(c, c, e)$. Clearly then $\mathrm{J}(\mathrm{r}(a), e)=e(a)$.

$\left(\mathrm{J}^{2} \Rightarrow \mathrm{K}\right)$ : First we show that $\left(\mathrm{J}^{2}\right)$ implies UIP. Clearly taking $C(x, y, u, v)=$ $\mathrm{I}(\mathrm{I}(A, x, y), u, v)$ we have $d(x)=\mathrm{r}(\mathrm{r}(x)): C(x, x, \mathrm{r}(x), \mathrm{r}(x))$. Hence for any $u, v$ : $\mathrm{I}(A, x, y)$,

$$
\mathrm{J}_{C, x, y}^{2}(u, v,(x) \mathrm{r}(\mathrm{r}(x))): \mathrm{I}(\mathrm{I}(A, x, y), u, v),
$$

that is, identity proofs are unique.

We construct K. Let $D(x, v)$ type $(x: A, v: \mathrm{I}(A, x, x))$ and suppose $d(x)$ : $D(x, \mathrm{r}(x)) \quad(x: A)$. Let $a: A$ and $c: \mathrm{I}(A, a, a)$. We have $\mathrm{J}_{C, a, a}^{2}(\mathrm{r}(a), c,(x) \mathrm{r}(\mathrm{r}(x)))$ : $\mathrm{I}(\mathrm{I}(A, a, a), \mathrm{r}(a), c)$ by $(11)$. Define $\mathrm{K}$ using reindexing (3):

$$
\mathrm{K}_{D, a}(c, d)={ }_{\text {def }} \mathrm{R}_{B, \mathrm{r}(a), c}\left(\mathrm{~J}_{C, a, a}^{2}(\mathrm{r}(a), c,(x) \mathrm{r}(\mathrm{r}(x))), d(a)\right): D(a, c)
$$

where $B(u)=D(a, u)$. Thus

$$
\mathrm{K}_{D, a}(\mathrm{r}(a), d)=\mathrm{R}_{B, \mathrm{r}(a), \mathrm{r}(a)}(\mathrm{r}(\mathrm{r}(a)), d(a))=d(a) .
$$

This shows the equivalence of the elimination rules.

Remark 7.2 However, also the $\mathrm{J}^{2}$-axiom suggested here breaks the standard mold of inductively defined sets and families in type theory, since the eliminated type $\mathrm{I}(A, a, b)$ occurs twice. It may though be seen as an axiomatization of a form of double elimination for identity types, of the kind derivable for simple inductive types like the unit type or the natural numbers. For explicit comparison, the unit type $\mathrm{N}_{1}$ with canonical element $0_{1}$ has the elimination rule

$$
\frac{c: \mathrm{N}_{1} \quad d: C\left(0_{1}\right)}{\mathrm{R}_{1, C}(c, d): C(c)}
$$


where $C(x)$ type for $x: \mathrm{N}_{1}$. From this we may derive a double elimination rule

$$
\frac{c: \mathrm{N}_{1} \quad c^{\prime}: \mathrm{N}_{1} \quad d: D\left(0_{1}, 0_{1}\right)}{\mathrm{R}_{1, D}^{2}\left(c, c^{\prime}, d\right): D\left(c, c^{\prime}\right)}
$$

where $D(x, y)$ type for $x: \mathrm{N}_{1}, y: \mathrm{N}_{1}$ with computation rule $\mathrm{R}_{1, D}^{2}\left(0_{1}, 0_{1}, d\right)=d$. The following construction fulfills the requirements

$$
\mathrm{R}_{1, D}^{2}\left(c, c^{\prime}, d\right)=_{\text {def }}\left(\mathrm{R}_{1, C}\left(c, \lambda y \cdot \mathrm{R}_{1, C^{\prime}}(y, d)\right)\right)\left(c^{\prime}\right)
$$

where $C(x)=\left(\Pi y: \mathrm{N}_{1}\right) D(x, y)$ and $C^{\prime}(y)=D\left(0_{1}, y\right)$.

\section{Concluding discussion}

We have seen that proof-relevant families of setoids appear in abundance in standard Martin-Löf type theory. Each family of types $B:(A)$ type yields such a family $\left(A^{*}, B^{*}\right)$. However this kind of families seems difficult to use for certain purposes, e.g. construction of categories with equality on objects. For such purposes the standard proof-irrelevant families are more suitable. They are, on the other hand, not easy to construct in standard type theory. Roughly speaking, it seems that we need to construct extensional collapses of the types involved in the families. This procedure is well-known from set theory, and indeed, one way of constructing such families is to use Aczel's method [1] for modelling constructive set theory CZF: apply the W-type construction to a universe of types and define equality of sets by W-recursion as bisimilarity of trees.

\section{References}

[1] Peter Aczel. The type-theoretic interpretation of constructive set theory. In: A. Macintyre, L. Pacholski and J. Paris (eds.), Logic Colloquium 'r7. North-Holland, Amsterdam 1978.

[2] Peter Aczel. Galois - a theory development project. Report for the 1993 Turin meeting on the Representation of Mathematics in Logical Frameworks. Available at URL: www.cs.man.ac.uk/ petera/papers.html

[3] Errett Bishop and Douglas S. Bridges. Constructive analysis. Springer 1985.

[4] Alexandre Buisse and Peter Dybjer. The interpretation of intuitionistic type theory in locally cartesian closed categories - an intuitionistic perspective, in Proceedings of MFPS XXIV, Philadelphia 2008. Electr. Notes Theor. Comput. Sci. 218(2008), $21-32$. 
[5] Richard Garner. Two-dimensional models of type theory. Mathematical Structures in Computer Science 19(2009), 687-736.

[6] Michael Hedberg. A coherence theorem for Martin-Löf's type theory. J. Funct. Programming 8 (1998), no. 4, 413-436.

[7] Martin Hofmann and Thomas Streicher. The groupoid interpretation of type theory. In: G. Sambin and J. Smith (eds.) Twenty-five years of constructive type theory (Venice, 1995), 83-111, Oxford Logic Guides, 36, Oxford Univ. Press, New York, 1998.

[8] Martin Hofmann and Thomas Streicher. The groupoid interpretation of type theory. Longer preprint version of [7]. URL: http://www2.tcs.ifi.lmu.de/ mhofmann/

[9] Ray Mines, Fred Richman and Wim Ruitenburg. A course on constructive algebra. Springer 1988.

[10] Ieke Moerdijk and Erik Palmgren. Type theories, toposes and constructive set theory: predicative aspects of AST, Annals of Pure and Applied Logic 114(2002), $155-201$.

[11] Bengt Nordström, Kent Peterson and Jan M. Smith. Programming in Martin-Löf type theory. Oxford University Press 1990.

[12] Thomas Streicher. Investigations into intensional type theory. Habilitation Thesis, Ludwig-Maximilians Universität, Munich 1993. www. mathematik.tu-darmstadt.de/ ${ }^{\sim}$ streicher/

ERIK PALMgRen

Department of Mathematics, Stockholm University, SE-106 91 Stockholm, Sweden

E-MAIL: palmgren@math.su.se

URL: www.math.su.se 